\title{
INTERVENCIÓN INTERACTIVA EN LOS PROBLEMAS DE COMPORTAMIENTO INFANTIL
}

\author{
Ariel Vite Sierra ${ }^{1 *}$, Javier Nahúm Alfaro Belmont ${ }^{1}$, Areli Dayanth Pérez Pérez ${ }^{1}$ y David Miranda García ${ }^{2}$ \\ ${ }^{1}$ Universidad Nacional Autónoma de México, ${ }^{2}$ Universidad Autónoma del Estado de México
}

Recibido, noviembre 26/2013

Concepto de evaluación, octubre 17/2014

Aceptado, noviembre 7/2014
Referencia: Vite Sierra, A., Alfaro Belmont, J.N., Pérez Pérez, A.D. \& Miranda García, D. (2015). Intervención interactiva en los problemas de comportamiento infantil. Acta Colombiana de Psicología, 18(1), 149-157. DOI: 10.14718/ACP.2015.18.1.14

Resumen

El objetivo del estudio fue examinar la efectividad de una estrategia de intervención con padres, basada en la perspectiva de la interacción social, a fin de disminuir los problemas de comportamiento infantil. Los participantes fueron 15 niños (11 niños y 4 niñas) con sus respectivas madres; las edades de los niños oscilaron entre los cinco y los ocho años, y el promedio de edad de las madres fue de 27.8 años. Se empleó un diseño experimental de caso único y se utilizaron procedimientos de enseñanza conductual como instrucciones, modelamiento, moldeamiento y retroalimentación visual. Se llevaron a cabo análisis de dependencias secuenciales y de secuencias temporales que son sensibles a los procesos de reforzamiento positivo y negativo que operan en las relaciones coercitivas. La intervención propició un aumento de la conducta prosocial en la madre y una disminución de la conducta aversiva infantil. Por otra parte, el cambio observado en la paternidad positiva parece mediar el cambio en los problemas de comportamiento infantil.

Palabras clave: problemas de conducta, coerción, interacción social, díadas

\section{INTERACTIONAL INTERVENTION IN CHILD BEHAVIOR PROBLEMS}

\begin{abstract}
The aim of the study was to examine the effectiveness of an intervention strategy with parents, based on the perspective of social interaction, in order to decrease child behavior problems. Participants were 15 children (eleven boys and four girls) with their mothers. Children ages ranged from five to eight years, and the average age of mothers was 27.8 years. A single case experimental design was used and behavioral teaching procedures such as instructions, modeling, shaping and visual feedback were applied. Analysis of sequential dependencies and time sequences sensitive to processes of positive and negative reinforcement operating in coercive relationships were carried out. The suitable intervention increased prosocial behavior in mothers and decreased aversive behavior in children. Moreover, the observed change in positive parenting appears to mediate the change in child behavior problems.

Key words: behavior problems, coercion, social interaction, dyads
\end{abstract}

\section{INTERVENÇÃO INTERATIVA NOS PROBLEMAS DE COMPORTAMENTO INFANTIL}

\begin{abstract}
Resumo
O objetivo do estudo foi examinar a efetividade de uma estratégia de intervenção com pais, baseada na perspectiva da interação social, a fim de diminuir os problemas de comportamento infantil. Os participantes foram 15 crianças (11 meninos e 4 meninas) com suas respectivas mães; as idades das crianças oscilaram entre os cinco e os oito anos, e a média de idade das mães foi de 27.8 anos. Empregou-se um desenho experimental de caso único e utilizaram-se procedimentos de ensino condutual como instruções, modelamento, modelagem e retroalimentação visual. Foram feitas análises de dependências sequenciais e de sequências temporais que são sensíveis aos processos de reforço positivo e negativo que operam nas relações coercitivas. A intervenção propiciou um aumento da conduta pró-social na mãe e uma diminuição da conduta aversiva infantil. Por outro lado, a mudança observada na paternidade positiva parece mediar a mudança nos problemas de comportamento infantil. Palavras chave: problemas de conduta, coerção, interação social.
\end{abstract}

* Facultad de Psicología, UNAM, Av. Universidad 3004, Col. Copilco-Universidad, C.P. 04510, Del. Coyoacán, México, D.F. avite@unam.mx - Tel. 55-56220555-41244 


\section{INTRODUCCIÓN}

Los problemas de comportamiento de niños y adolescentes representan un problema común, grave y oneroso. Un análisis económico mostró que los costos combinados de atención de la salud, servicios sociales y educación representan un incremento de hasta diez veces más para los niños con trastorno de conducta en relación con los que no lo presentan (Furlong, McGilloway, Bywater, Hutchings, Smith, \& Donnelly, 2012).

En 2006 el National Institute for Health and Care Excellence (NICE, por sus siglas en inglés) afirmaba que la prevalencia de los trastornos de conducta en niños de cinco a diez años era de $6,9 \%$ en niños y de $2,8 \%$ en niñas, de los cuales, el 4,5\% de los niños y el 2,4\% de las niñas mostraban rasgos negativistas-desafiantes; estableciéndose como los problemas más comunes en edad infantil por los que los padres generalmente solicitan intervención profesional (Egger \& Angold, 2006).

Asimismo, se ha señalado la existencia de una trayectoria de desarrollo de los problemas de comportamiento: estos surgen en la edad preescolar, continúan en la primaria, tienden a incrementarse en frecuencia y gravedad a través de la adolescencia, y probablemente desembocan en problemas de conducta antisocial en la adultez (Timmermans, Van Lier, \& Koot, 2010; Webster-Stratton, Rinaldi, \& Reid, 2011).

Un ejemplo de ello es el estudio longitudinal de Pihlakoski, Sourander, Aromaa, Rautava et al. (2006), quienes desde un marco conductual (Achenbach, 1978) señala que los problemas externalizantes se mantienen e incrementan de los tres a los 12 años, mientras que los problemas internalizantes surgen en la pre-adolescencia. Considerando a los problemas externalizantes como los predictores de un gran número de psicopatologías en etapas posteriores del desarrollo, de manera particular la agresión muestra estabilidad a través del tiempo y del género, así como los problemas de origen somático.

Por otra parte, se ha señalado que los problemas de comportamiento infantil tienden a desarrollarse en el contexto de una paternidad severa e inconsistente, y bajos niveles de crianza positiva (Gardner, Ward, Burton, \& Wilson, 2003), lo que sitúa a los niños en alto riesgo de problemas posteriores, incluyendo la exclusión escolar, la delincuencia, las enfermedades mentales, la violencia de pareja, falta de alfabetización, el desempleo y la mortalidad elevada (Fergusson, Horwood, \& Ridder, 2005; Romero, Robles, \& Lorenzo, 2006).

Para comprender mejor las causas de los problemas de comportamiento, los teóricos a menudo apuntan a la importancia de los períodos de desarrollo temprano. Por ejemplo, las teorías interaccionales vinculan la calidad de los intercambios sociales entre padres e hijos durante la primera infancia con las relaciones interpersonales y el ajuste del comportamiento infantil en la infancia y la adolescencia (Shaw \& Bell, 1993). Las interacciones entre padres e hijos que se caracterizan por una intensa hostilidad y emocionalidad negativa promueven ciclos de interacción coercitiva a través de un proceso de reforzamiento (Patterson, 1982).

De manera particular, la hipótesis de la coerción (Patterson, 1982) describe un patrón de interacción entre los padres y el niño. En este modelo la disciplina rígida es escalada en el tiempo y es reforzada por medio de obediencia infantil a corto plazo. Debido a la inseguridad y al miedo propiciado por una paternidad inconsistente y aversiva, el niño evita las instrucciones parentales y frecuentemente modela la conducta negativa. Esta teoría señala que un incremento de las conductas de evitación y agresión del niño funcionan como disparadores para una mayor disciplina rígida, por lo que este tipo de interacción se estabiliza con el paso del tiempo, llegando a regular y fomentar una interacción hostil y peligrosa (Hakman, Chaffin, Funderbunk, \& Silovsky, 2009; Timmer, Urquiza, Zebell, \& McGrath, 2005).

La importancia del desarrollo de intercambios coercitivos entre padres e hijos puede no manifestarse hasta que los niños ingresan a la escuela. Compañeros y maestros responden a la conducta externalizante del niño con resistencia y rechazo, lo que lleva a una serie de problemas en cascada durante la niñez y la adolescencia. Por lo tanto, la calidad de las relaciones entre padres e hijos en la primera infancia tiene importancia evolutiva en virtud de que los niños aprenden estrategias para interactuar con otros que afectan su comportamiento y relaciones futuras (Berkien, Louwerse, Verhulst, \& van der Ende, 2012, Cunningham \& Ollendick, 2010; Furlong et al. 2012).

Basados en la teoría de la coerción, se ha desarrollado un gran número de intervenciones con padres en los últimos años, las cuales tienen como objetivo el restructurar los patrones interactivos madre-hijo a fin de promover el comportamiento prosocial infantil y disminuir las conductas antisociales u oposicionales. A la fecha existe una amplia evidencia empírica que muestra su eficacia (Capaldi, Pears, Kerr, Owen, \& Kim, 2012; Patterson, DeGarmo, \& Forgatch, 2004; Reed, Snyder, et al. 2013; Webster-Stratton \& Reid, 2003).

Considerando los señalamientos anteriores, el objetivo del estudio es poner a prueba la efectividad de una estrategia basada en la perspectiva de la interacción social para abordar los problemas de comportamiento infantil. 


\section{MÉTODO}

\section{Participantes}

Participaron 15 niños (11 niños y 4 niñas) con sus respectivas madres, cuyas edades oscilaron entre los cinco y ocho años, y que fueron canalizados para tratamiento por el equipo psicopedagógico de diferentes escuelas primarias del sur de la ciudad de México. El promedio de edad de las madres fue de 27.8 años, con una escolaridad media de primaria y de nivel socioeconómico bajo.

\section{Instrumentos}

Entrevista de Evaluación Psicosituacional. Tiene como propósito conocer las características conductuales de la problemática que presenta la díada. Dicha entrevista está dividida en seis partes: a) determinación de la conducta objetivo, b) descripción de situaciones y ambientes específicos en los que ocurre la conducta, c) contingencias que estimulan y mantienen la conducta, d) determinación de la relación de interacción entre el niño y los padres, e) métodos empleados para el control de la conducta y f) detección de ideas irracionales (Walker \& Shea, 1987).

Inventario de Comportamiento Infantil (Eyberg \& Ross, 1978). Consta de 36 reactivos que evalúan la conducta del niño en los últimos tres meses, donde los padres tienen la posibilidad de elegir en una escala tipo Likert de tres puntos $(1=$ nunca, $2=$ a veces y $3=$ siempre $)$ la frecuencia con la cual los niños exhiben ciertas conductas, además de dos opciones de respuesta (Si o No) a la pregunta: ¿representa un problema? Para este estudio se utilizó la escala adaptada a población mexicana, la cual cuenta con un índice de confiabilidad de Cronbach de 0.90 para la subescala de frecuencia y de 0.91 para la subescala de "representa un problema"; la confiabilidad global de la escala es de 0.90 (Vite, Negrete, \& Miranda, 2012).

Sistema de Captura de Datos Observacionales (Vite, García, \& Rosas, 2005). Es un sistema computarizado de registro observacional para el estudio de la interacción madre-niño en ambientes controlados, y comprende un conjunto de categorías de comportamiento, mutuamente excluyentes y exhaustivas, de la madre y del niño. Sus principales características son; a) permite un registro continuo de las interacciones, b) posibilita la codificación secuencial de eventos, c) permite la obtención de medidas de frecuencia por sesión de cada código, tanto de la madre como del hijo, y d) permite registrar sus tasas de respuesta por minuto.
Las conductas a evaluar fueron las siguientes clases de respuestas:

- Comportamiento prosocial infantil: inclúa las conductas de aproximación social, obedecer, petición y realizar actividad.

- Comportamiento aversivo infantil: abarcaba las conductas de desobedecer, repelar y quejarse.

- Paternidad positiva: incluía las conductas de aprobación, reflejo, atención social, obediencia y elogio.

- Paternidad negativa: comprendía las conductas de rehusarse, amenazar, desaprobar y regañar.

\section{Procedimiento}

Como primer paso se aplicó la entrevista psicosituacional a fin de recabar información sobre la problemática reportada. A continuación se solicitó a cada una de las madres la firma del Consentimiento Informado. Posteriormente se llevaron a cabo las fases de línea base, intervención y seguimiento.

Linea base. El total de observaciones de la interacción de las díadas madre-hijo, se llevó a cabo en tres sesiones de 30 minutos, en las cuales se realizaba una tarea de tipo académico, que se estructuró de acuerdo con el nivel de escolaridad de cada niño.

Intervención. Las primeras sesiones de esta fase se orientaron a enseñar a las madres cómo proporcionar de manera contingente al comportamiento social infantil (aproximaciones sociales, obedecer, realizar la actividad) atención positiva consistente en aproximaciones sociales, obedecer, elogiar y reflejar, así como aprobación de las conductas de obediencia.

De manera particular, el experimentador modelaba las conductas de interés, señalando las características destacadas para su ejecución. Acto seguido, se solicitaba a la madre que ensayara la realización de dichas conductas con su hijo, desarrollando la tarea escolar. La sesión finalizaba proporcionando retroalimentación sobre la ejecución de la madre. En las sesiones subsiguientes, nuevamente se le mostraba a las madres segmentos de interacciones con sus hijos, en donde se subrayaba el empleo apropiado e inapropiado de las conductas de aproximación social, obedecer, aprobación, elogiar y reflejar, siguiendo la misma dinámica de las primeras sesiones.

Seguimiento. Esta fase se llevó a cabo un mes después de concluir la intervención y consistió en tres sesiones, una cada semana, en las cuales no se proporcionó instrucción o retroalimentación alguna a las madres al realizar la tarea escolar con sus hijos, y se aplicó a éstas el Inventario 
de comportamiento infantil con la finalidad de evaluar la frecuencia de las conductas problemáticas en sus hijos.

\section{RESULTADOS}

Los resultados obtenidos se presentan en el siguiente orden: en primer lugar, el índice de concordancia entre observadores; en segunda instancia, los datos del Inventario de comportamiento infantil antes y después de la intervención; posteriormente se presenta el análisis descriptivo en frecuencias de las conductas de interés en la línea base y el seguimiento. En cuarto lugar, los datos del análisis de los episodios de comportamiento infantil y materno en las fases de línea base y seguimiento, y finalmente, los datos generados por el análisis de regresión múltiple entre tratamiento y paternidad positiva con el comportamiento aversivo infantil.

\section{Concordancia}

Se seleccionó al azar un 30\% del total de sesiones de observación de las interacciones madre-hijo; posteriormente, dos observadores independientes codificaron las filmaciones. En consonancia con Hartmann (1977) se decidió obtener el acuerdo de las cinco variables que fueron empleadas en el análisis de datos, lo cual se muestra en la tabla 1.
En la tabla 2 se muestran los resultados obtenidos a través del Inventario de comportamiento infantil. En la línea base, las madres reportan una alta ocurrencia de conductas inadecuadas (79.7 de 108 pts.), y su percepción de que éstas representan un problema para ellas (25 de 36 pts.). En el seguimiento, la frecuencia de las conductas disminuye (49.4 de 108 pts.), así como la percepción de que éstas representan un problema (4.7 de 36 pts.).

Para el análisis de los datos se llevó a cabo el siguiente proceso: los archivos en tiempo real fueron transformados en secuencias de comportamiento, de acuerdo con un muestreo temporal segundo a segundo. Posteriormente, los archivos de datos de la madre y del niño se transformaron en uno solo, de forma que una secuencia de comportamiento fue la variable correspondiente a la madre y otra, al hijo. Los datos resultantes se analizaron a través del paquete estadístico Statistica, a fin de obtener las frecuencias de las madres y sus hijos en las diferentes categorías conductuales consideradas para el estudio, con el propósito de determinar los cambios significativos entre la línea base y el seguimiento. Con los valores obtenidos se aplicó la prueba no paramétrica de pares de Wilcoxon, para cada una de las categorías de interés tanto de las madres como de los niños.

La tabla 3 muestra los datos de las conductas de los niños en las fases de línea base y seguimiento. Como se aprecia,

Tabla 1.

Índice de concordancia de las conductas Maternidad positiva, Maternidad negativa, Conducta prosocial infantil y Conducta aversiva infantil en las fases de línea base y mantenimiento.

\begin{tabular}{ccccc}
\hline \multicolumn{1}{c}{ Fases } & $\begin{array}{c}\text { Paternidad } \\
\text { positiva }\end{array}$ & $\begin{array}{c}\text { Paternidad } \\
\text { negativa }\end{array}$ & $\begin{array}{c}\text { Conducta prosocial } \\
\text { infantil }\end{array}$ & $\begin{array}{c}\text { Conducta aversiva } \\
\text { infantil }\end{array}$ \\
\hline Línea base & .88 & .90 & .79 & .89 \\
Seguimiento & .90 & .95 & .95 & .91 \\
\hline
\end{tabular}

Nota. Valores de Kappa: de $.40-.60=$ aceptable, de $.60-075=$ buenos y mayor a $.75=$ excelente.

Tabla 2

Resultados de la aplicación del Inventario de Comportamiento Infantil en la línea base y el seguimiento.

\begin{tabular}{lcccc}
\hline & Línea & Base & Tratamiento & \\
\hline \multirow{3}{*}{ Frecuencia } & $\mathrm{M}$ & $\mathrm{DE}$ & $\mathrm{M}$ & $\mathrm{DE}$ \\
Problema & 79.71 & 69.12 & 25.28 & 18.19 \\
& 25.67 & 21.52 & 9.87 & 7.65 \\
\hline
\end{tabular}


la conducta prosocial (aproximación social, obedecer, petición y realizar la actividad) se presentó con un promedio de 89.79 en la línea base y de 106.71 durante el seguimiento, siendo la diferencia estadísticamente significativa $(\mathrm{z}=182.83 \mathrm{p}=<.00)$. En lo que respecta al comportamiento aversivo (desobedecer, repelar y quejarse) este tuvo un promedio en la línea base de 66.78 y de 19.09 en la fase de seguimiento, mostrando una disminución estadísticamente significativa $(\mathrm{z}=199.15 \mathrm{p}=<.00)$.

Con respecto al comportamiento materno, en la tabla 4 se muestran los datos de la línea base y seguimiento. Como se observa, el promedio de la conducta de paternidad positiva en la línea base fue de 109.78 y de 136.76 en el seguimiento, mostrando un cambio estadísticamente significativo $(\mathrm{z}=205.48 \mathrm{p}=<.00)$. En lo referente a la conducta de paternidad negativa su porcentaje promedio durante la línea base fue de10.69 y de 4.34 en el seguimiento, con una disminución estadísticamente significativa $(\mathrm{z}=229.07 \mathrm{p}=<.00)$.
Con el fin de probar si la crianza positiva observada es un mecanismo de cambio en el tratamiento, se llevaron a cabo, de manera inicial, las correlaciones entre el tratamiento y el comportamiento negativo del niño $(r=0,40$, $\mathrm{p}=0,001)$, entre el tratamiento y la crianza positiva $(r=0,27, p=0,00)$, $y$ entre la crianza y el comportamiento negativo del niño $(r=0,35, p=0,00)$.

En segundo lugar, se llevó a cabo un análisis de regresión múltiple jerárquica, en el cual se consideró el cambio del comportamiento infantil negativo como DV. La tabla 4 muestra cómo se introdujeron las variables independientes: En primer lugar, se introdujo el tratamiento como posible predictor de la conducta aversiva infantil, obteniendo una predicción estadísticamente significativa $(\mathrm{R} 2=.35$, $\mathrm{F}(258.15)=1.53, \mathrm{p}<00)$. En segundo lugar, se introdujeron como posibles variables predictoras el tratamiento y la paternidad positiva, obteniendo una predicción estadísticamente significativa $(\mathrm{R} 2=.42, \mathrm{~F}(242.97)=2.53, \mathrm{p}<.00)$.

Tabla 3.

Porcentaje promedio de episodios de comportamiento infantil en las fases de línea base y seguimiento.

\begin{tabular}{lccccc}
\hline & Línea & Base & & Tratamiento & \\
\hline & $\mathrm{M}$ & $\mathrm{DE}$ & $\mathrm{M}$ & $\mathrm{DE}$ & $\mathrm{P}$ \\
\hline $\begin{array}{l}\text { Comportamiento prosocial } \\
\begin{array}{l}\text { (Aproximación social, obedecer, } \\
\text { petición y realizar actividad) }\end{array}\end{array}$ & 89.79 & 78.94 & 106.71 & 99.21 & .00 \\
$\begin{array}{l}\text { Comportamiento aversivo } \\
\text { (Desobedecer, repelar y quejarse) }\end{array}$ & 66.78 & 56.21 & 19.09 & 16.65 & .00 \\
\hline
\end{tabular}

Tabla 4.

Porcentaje promedio de episodios del comportamiento materno en las fases de línea base y seguimiento.

\begin{tabular}{llllcc}
\hline & Línea & Base & & Tratamiento & \\
\hline & $\mathrm{M}$ & $\mathrm{DE}$ & $\mathrm{M}$ & $\mathrm{DE}$ & $\mathrm{p}$ \\
\hline $\begin{array}{l}\text { Paternidad Positiva } \\
\text { (Aproximación social, obedecer, } \\
\text { aprobar, elogiar y reflejar) }\end{array}$ & 109.78 & & 136.76 & & .00 \\
$\begin{array}{l}\text { Paternidad Negativa } \\
\text { (Rehusar, amenazar, desaprobar } \\
\text { y regañar) }\end{array}$ & 10.69 & 9.76 & 4.34 & 3.23 & .00 \\
\hline
\end{tabular}


Tabla 5.

Modelo de regresión múltiple entre Tratamiento y Paternidad Positiva con el comportamiento aversivo infantil.

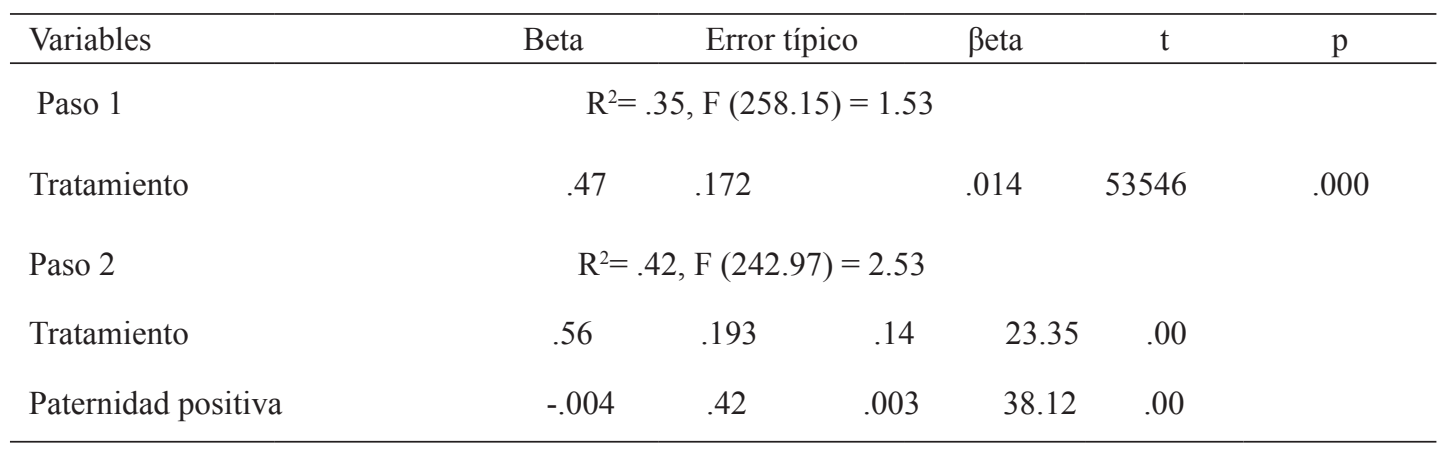

\section{DISCUSIÓN}

El interés principal del estudio fue poner a prueba una estrategia interaccional para decrementar los problemas de comportamiento infantil. Los resultados obtenidos señalan que cuando las madres son sensibles y exhiben tasas globales de prácticas parentales positivas, se favorece de manera general la conducta prosocial infantil y, de manera particular, la obediencia. Estos resultados, son similares a los estudios descritos en la literatura (Herruzo, Pino, \& Raya, 2009; Kochanska \& Aksan, 2006; Vite \& López, 2007).

De manera particular, se observa una disminución sustancial de la frecuencia de los problemas de conducta infantil, aunada a la disminución de conductas maternas aversivas (especialmente desaprobar y regañar), y el incremento de comportamientos prosociales (principalmente aprobar, elogiar y reflejar).

Además, se muestra que el cambio en las prácticas parentales parece ser parcial y significativo como mediador en el cambio del comportamiento de los niños. Estos datos son sustanciales ya que proporcionan una prueba directa de la noción de que el cambio de esta habilidad puede ser el ingrediente más destacado en los programas para una crianza efectiva (Hutchings, Lane \& Gardner, 2004). Los estudios sobre los efectos de mediación han sido poco frecuentes (Rutter, 2005), pero son vitales para la comprensión de los procesos de tratamiento subyacentes y los factores causales de la conducta del niño.

Estos resultados se suman a un cuerpo de trabajo que sugiere que la crianza positiva puede desempeñar un papel causal en el desarrollo y el cambio en la conducta de los niños problema (Gardner et al. 2003).

Asimismo, los datos generados están en línea con la teoría de la coerción de Patterson (1982), que establece que una secuencia de interacciones sobre la base del reforza- miento negativo mantiene los problemas de comportamiento agresivo en los niños. Esta secuencia comienza cuando una madre actúa de manera aversiva hacia el niño, el niño reacciona aversivamente a la madre, y la madre cede. La conducta del niño es, por lo tanto, reforzada y es probable que se incremente en el futuro.

Esto confirma los resultados de la investigación experimental previa que ha mostrado los efectos de los niños sobre los padres (por ejemplo, Pelham et al. 1997) así como como los efectos de los padres sobre los niños (WebsterStratton \& Taylor, 2001). Las relaciones recíprocas manifestadas en el presente estudio son consistentes con estudios longitudinal anteriores (Gardner et al. 2003; Gross, Shaw, Moilanen, Dishion, \& Wilson, 2008) que han apoyado modelos recíprocos de funcionamiento entre padres e hijos (Harvey \& Metcalfe, 2012; Patterson, et al. 2004).

Por otra parte, la enseñanza a las madres de conductas como el empleo del elogio, el cual está relacionado con el reconocimiento de las capacidades, características y aptitudes del niño, y que cumple el papel de reforzador y motivador, aumentó la probabilidad de ocurrencia de la conducta prosocial, incluida la obediencia (Strand, 2000). Asimismo, el uso de la retroalimentación materna a las conductas adecuadas del niño propicia dos patrones interactivos: a) cuando el niño realiza la actividad académica la madre responde con aprobaciones, generando así un patrón sincrónico; y b) cuando la madre emplea reflejos, el niño responde con comportamientos prosociales.

Lo anterior revela cómo las interacciones recíprocas madre-niño fomentan el desarrollo de conductas prosociales infantiles, resaltando la importancia de las contingencias sociales orquestadas por la madre y que propician a su vez la obediencia infantil (Strand, Wahler, \& Herring, 2001; Wahler, Castellani, Smith, \& Kahtley, 1996). 
Estas reacciones "apropiadas y oportunas" han sido caracterizadas por los investigadores bajo el rubro de prácticas paternales apropiadas (Kochanska, Aksan, \& Nichols, 2003; Strand et al. 2001). De manera particular, son respuestas paternales específicas que contienen aprobaciones y/o información selectiva (reflejo), que es contingente a la conducta del menor después de su conducta (Shelton, Frick, \& Wooten, 1996; Wahler \& Meginnis, 1997).

$\mathrm{Al}$ respecto, se ha señalado que la crianza de los hijos caracterizada por bajos niveles de calidez, apoyo y participación interfiere con la capacidad de un niño para modular y regular su excitación, y como resultado, un niño puede ser menos capaz de considerar las consecuencias de sus acciones y abstenerse de exhibir conductas agresivas (Brody, Dorsey, Forehand, \& Armistead, 2002).

Los resultados de este estudio tienen que considerarse a la luz de una serie de limitaciones. En primer lugar, con respecto al Inventario de comportamiento infantil los datos señalan que la media del percentil fue 79.71, lo que indica que el grupo de niños se encontraba en situación de riesgo para los trastornos de conducta. Por lo que es posible que se incluyera una serie de falsos positivos (Offord \& Bennett, 2002), y esto pudo tener un efecto sobre el resultado de la intervención. No obstante, la identificación precisa de los niños en situación de riesgo para el desarrollo de un patrón crónico de problemas de conducta es esencial para las intervenciones de prevención eficaces (Hill, Coie, Lochman, \& Greenberg, 2004).

En segundo lugar, los resultados podrían estar sesgados por el nivel educativo bajo de los padres que participaron en el presente estudio. Por lo tanto, estos hallazgos tienen una limitada generalización para padres con otros niveles educativos.

Y en tercer lugar, a pesar de que el presente estudio proporciona evidencia de cambios en las habilidades de crianza de las madres y el comportamiento infantil como resultado de la estrategia de intervención empleada, así como el efecto mediador de las prácticas de crianza en los problemas de conducta infantil, y que se realizó un seguimiento después de un mes, no se pueden establecer conclusiones con respecto a los efectos a largo plazo de la estrategia de intervención.

Considerando que este estudio mostró efectos sobre los problemas de comportamiento en el seguimiento a un mes y un efecto de mediación de las prácticas parentales apropiadas sobre el comportamiento inapropiado del niño, se considera pertinente desarrollar estudios en donde se determine, como una dimensión continua (por ejemplo, control psicológico, control conductual, calidez/apoyo), la relación precisa de las prácticas parentales positivas en los problemas de comportamiento infantil.

\section{REFERENCIAS}

Achenbach, T. M. (1978). The Child Profile: I. Boys age 6-11. Journal of Consulting and Clinical Psychology, 46, 478-488.

Berkien, M., Louwerse, A., Verhulst, F., \& van der Ende, J. (2012). Children's Perceptions of Dissimilarity in Parenting Styles are Associated with Internalizing and Externalizing Behaviour. European Child and Adolescent Psychiatry, 21, 79-85.

Brody, G. H., Dorsey, S., Forehand, R., \& Armistead, L. (2002). Unique and Protective Contributions of Parenting and Classroom Processes to the Adjustment of Africa American Children Living in Single-Parent Families. Child Development, 73, 274-286.

Capaldi, D. M., Pears, K. C., Kerr, D. C., Owen, L. D., \& Kim, H. K. (2012). Growth in Externalizing and Internalizing Problems in Childhood: A Prospective Study of Psychopathology across Three Generations. Child Development, 83, 1945-1959.

Cunningham, N. R., \& Ollendick, T. H. (2010). Comorbidity of Anxiety and Conduct Problems in Children: Implications for Clinical Research and Practice. Clinical Child and Family Psychology Review, 13, 333-347.

Egger, H. L., \& Angold, A. (2006). Common Emotional and Behavioral Disorders in Preschool Children: Presentation, Nosology, and Epidemiology. Journal of Child Psychology and Psychiatry, 47, 313-337.

Eyberg S.M., \& Ross A. W. (1978). Assessment of Child Behavior: The Validation of a New Inventory. Journal of Clinical Child Psychology, 7, 113-116.

Fergusson, D.D., Horwood, L.J., \& Ridder, E. (2005). Show me the Child at Seven: The Consequences of Conduct Problems in Childhood for Psychosocial Functioning in Adulthood. Journal of Child Psychology and Psychiatry, 46, 837-49.

Furlong, M., McGilloway, S., Bywater, T., Hutchings, J., Smith, S. M., \& Donnelly, M. (2012). Behavioural and CognitiveBehavioural Group-Based Parenting Programmes for Early-Onset Conduct Problems in Children Aged 3 to 12 Years (Review). The Cochrane Collaboration 2, 1-357.

Gardner, F., Ward, S., Burton, J., \& Wilson, C. (2003). The Role of Mother-Child Joint Play in the Early Development of Children's Conducts Problems: A Longitudinal Observational Study. Social Development, 12, 361-379.

Gross, H. E., Shaw, D. S., Moilanen, K. L., Dishion, T. J., \& Wilson, M. N. (2008). Reciprocal Models of Child Behavior and Depressive Symptoms in Mothers and Fathers in a Sample of Children at Risk for Early Conduct Problems. Journal of Family Psychology, 22, 742-751.

Hakman, M., Chaffin, M., Funderbunk, B., \& Silovsky, J. (2009). Change Trajectories for Parent-Child Interaction Sequences during Parent-Child Interaction Therapy for Child Physical Abuse. Child Abuse \& Neglect, 33, 461-470.

Hartmann, D. P. (1977). Considerations in the Choice of Interobserver Reliability Estimates. Journal of Applied Behavior Analysis, 10, 103-116. 
Harvey, E. A. \& Metcalfe, L. A. (2012). The Interplay Among Preschool Child and Family Factors and the Development of ODD symptoms, Journal of Clinical Child \& Adolescent Psychology, 41, 458-470.

Herruzo, J., Pino, J. \& Raya, A. (2009) La agresividad en la infancia: El estilo de crianza parental como factor relacionado. European Journal of Education and Psychology, 2, 211-222.

Hill, L. G., Coie, J. D., Lochman, J. E., \& Greenberg, M. T. (2004). Effectiveness of Early Screening for Externalizing Problems: Issues of Screening Accuracy and Utility. Journal of Consulting and Clinical Psychology, 72, 809-820.

Hutchings, J., Lane, E., \& Gardner, F. (2004). Making Evidence-Based Interventions Work. In D. Farrington, C. Sutton, \& D. Utting (Eds.), Support from the Start: Working with Young Children and Families to Reduce Risks of Crime and Antisocial Behaviour. London: DFES.

Kochanska, G., Aksan, N., \& Nichols, K. E. (2003). Maternal Power Assertion in Discipline and Moral Discourse Contexts: Commonalities, Differences, and Implications for Children's Moral Conduct and Cognition. Developmental Psychology, 39, 949-963.

Kochanska, G., \& Aksan, N. (2006). Children's Conscience and Self-Regulation. Journal of Personality, 74, 1588-1618.

National Institute for Health and Care Excellence (2006). Parent Training/Education Programs in the Management of Children with Conduct Disorders. www.nice.org.uk/TA102

Offord, D. R., \& Bennett, K. J. (2002). Prevention. In M. Rutter \& E. Taylor (Eds.), Child and Adolescent Psychiatry (4th Ed.) (pp. 881-899). Oxford, UK: Blackwell Science.

Patterson, G. (1982). Coercive family process. Eugene, OR: Castalia.

Patterson, G., DeGarmo, D., \& Forgatch, M. S. (2004). Systematic Changes in Families Following Prevention Trials. Journal of Abnormal Child Psychology, 32, 621-633.

Pelham, W., Lang, A. R., Atkeson, B., Murphy, D. A., Gnagy, E. M., Greiner, A. R. Greenslade, K. E. (1997). Effects of Deviant Child Behavior on Parental Distress and Alcohol Consumption in Laboratory Interactions. Journal of Abnormal Child Psychology, 25, 413-424.

Pihlakoski L, Sourander A, Aromaa M, Rautava P., et al. (2006). The Continuity of Psychopathology from Early Childhood to Preadolescence: A Prospective Cohort Study of 3-12-Year-Old Children. European Child and Adolescent Psychiatry, 15, 409-417.

Reed, A., Snyder, J., Staats, S., Forgatch, M. S., DeGarmo, D. S., Patterson, G. R., Low, S., Sinclair, R., \& Schmidt, N. (2013). Duration and Mutual Entrainment of Changes in Parenting Practices Engendered by Behavioral Parent Training Targeting Recently Separated Mothers. Journal of Family Psychology, 27(3), 343-354.

Romero, E., Robles, Z., \& Lorenzo, E. (2006). Prácticas parentales, atmósfera familiar y problemas de conducta externalizante en la infancia. Revista de Psiquiatría de la Facultad de Medicina de Barcelona, 33, 84-92.

Rutter, M. (2005). Environmentally Mediated Risks for Psychopathology: Research Strategies and Findings. Journal of the American Academy of Child and Adolescent Psychiatry, 44, 3-18.

Shaw, D. S., \& Bell, R. Q. (1993). Developmental Theories of Parental Contributors to Antisocial Behavior. Journal of Abnormal Child Psychology, 21, 493-518.

Shelton, K. K., Frick, P. J., \& Wooten, J. (1996). Assessment of Parenting Practices in Families of Elementary SchoolAge Children. Journal of Clinical Child Psychology, 25, 317-329.

Strand, P. S. (2000). A Modern Behavioral Perspective on Child Conduct Disorder: Integrating Behavioral Momentum and Matching Theory. Clinical Psychology Review, 20, 593-61.

Strand, P. S. Wahler, R. G., \& Herring, M. (2001). BehaviorSpecific Versus and Behavior-Nonspecific Reinforcement and Child Responses to Mother Instructions. Behaviour Research and Therapy, 39, 1085-1097.

Timmer, S.G., Urquiza, A.J. Zebell, M.N., \& McGrath, J.M. (2005). Parent-Child Interaction Therapy: Application to Maltreating Parent-Child Dyads. Child Abuse \& Neglect, $29,825-842$.

Timmermans, M. Van Lier. P.C., \& Koot, H.M. (2010). The Role of Stressful Events in the Development of Behavioural and Emotional Problems from Early Childhood to Late Adolescence. Psychological Medicine, 40, 1659-1668.

Vite, A, García, R., \& Rosas, C. (2005). Sistema de captura de datos observacionales SOI-I. Facultad de Psicología, UNAM.

Vite, A., Negrete, A., \& Miranda, D. (2012). Relación mediacional de los esquemas cognitivos maternos en los problemas de comportamiento infantil. Psicología y Salud, 22, 27-36.

Vite, A., \& López, F. (2007). La regulación aversiva en las interacciones madre-niño en díadas con historia de maltrato infantil. Apuntes de Psicología, 25, 145-156.

Wahler, R. G., \& Meginins, K. L. (1997). Strengthening Child Compliance through Positive Parenting Practices: What Works? Journal of Clinical Child Psychology, 26, 433-440. Journal of Clinical Child Psychology, 26, 433-440.

Wahler, R. G., Castellani, M. E., Smith, G. D., \& Keathley, E. A. (1996). Solitary Behavior and Friendly Social Activity: Differential Gateways for Conduct Problem versus Normal Child-Mother Dyads. Journal of Clinical Child Psychology, 25, 238-245.

Walker, J. E., \& Shea, T. M. (1987). Manejo Conductual. México: Manual Moderno.

Webster-Stratton, C., \& Taylor, T. (2001). Nipping Early Risk Factors in The Bud: Preventing Substance Abuse, Delinquency, and Violence in Adolescence Through 
Interventions Targeted at Young Children (0 to 8 years). Prevention Science, 2, 165-192.

Webster-Stratton, C., \& Reid, M. J. (2003). The IY Parents, Teachers, and Children Training Series. In A. Kazdin, \& J. Weisz (Eds.), Evidence-based psychotherapies for children and adolescents (pp. 224-240). New York: The Guilford Press.
Webster-Stratton, C., Rinaldi, J., \&. Reid, J. M. (2011). Longterm Outcomes of Incredible Years Parenting Program: Predictors of Adolescent Adjustment. Child and Adolescent Mental Health, 16, 38-46. 